\title{
Ribbed iron-based shape memory alloy bars for pre-stressed strengthening applications
}

\author{
Bernhard Schranz ${ }^{1,2}$ \\ Christoph Czaderski ${ }^{1}$, Moslem Shahverdi ${ }^{1,3}$, Julien Michels ${ }^{4}$, Thomas Vogel ${ }^{2}$, Masoud Motavalli ${ }^{1,3}$ \\ ${ }^{1}$ Structural Engineering Research Laboratory, Empa, Dübendorf, Switzerland \\ ${ }^{2}$ Institute of Structural Engineering (IBK), ETH Zürich, Switzerland \\ ${ }^{3}$ School of Civil Engineering, University of Tehran, Iran \\ ${ }^{4}$ re-fer AG, Brunnen, Switzerland
}

Contacting author: moslem.shahverdi@empa.ch

\section{Abstract}

Iron-based shape memory alloys (Fe-SMA) are a cost-effective alternative to conventional strengthening materials. With their unique properties such as the shape-memory effect (SME), structures can be retrofitted regarding the serviceability state as well as ultimate load capacity. The term SME refers to the ability of the material to return to its original shape upon heating, after having been deformed at ambient temperature. The heating and cooling process are generally referred to as "activation" of the alloy. If the material is restrained at activation, the material is subjected to tensile stresses. This phenomenon can be used for structural pre-stressing. The Fe-SMA material can be produced in form of standard-geometry reinforcement bars, to be used for flexural as well as shear-strengthening of concrete members. As a base for novel strengthening methods, this study aims at investigating the thermo-mechanical behaviour of FeSMA bars under realistic conditions. It comprises loading the material after activation (pre-stressed), exposure to multi-cyclic loading and observation of relaxation behaviour of pre-stressed Fe-SMA bars. Experiments were performed with diameter 16 and $12 \mathrm{~mm}$ bars. The results show a highly non-linear stress-strain behaviour, unlike conventional pre-stressing materials. The experiments with activated samples show that the stress-strain curve of Fe-SMA bars loaded after activation will approach and then follow the curve of non-activated tensile tests approximately in the point of the initial deformation. Furthermore, analytical material models are developed which show good correspondence with experimental data and serve as a base for further studies and structural design.

Keywords: Iron-based, shape memory alloy, bars, prestressing, strengthening, concrete structures

\section{Introduction}

The iron-based shape memory alloy (Fe-SMA), developed at Empa, the Swiss Federal Laboratories for Materials Science and Technology in Dübendorf, Switzerland [1] is an effective option to conventional strengthening materials. It can be used for upgrading the structural behaviour of concrete [2-6], as well as metallic structures [7]. The material offers unique properties that can be utilized in structural applications, most importantly the shape-memory effect (SME) [8]. The term SME refers to the ability to return to its originals shape when heated, after having been deformed at ambient temperature. The heating and cooling process in this context is called activation. The SME originates from a phase transformation in the lattice structure between martensite and austenite [9]. If the material is fixed against deformation when activated, tensile stresses develop in the respective direction, which is referred to as recovery stress. This phenomenon can be utilized to pre-stress structural members.

The material is produced in the form of standardgeometry reinforcement bars at industrial scale by the provider re-fer AG from Brunnen, Switzerland. 
The material properties of diameter $16 \mathrm{~mm}$ bars were examined in [3]. Besides standard testing procedures, it is essential to gain knowledge about the mechanical behaviour under realistic conditions. This requires assessing the quasi-static and cyclic stress-strain behaviour after restrained activation. Similar investigations were performed with Fe-SMA strips in $[6,7,10]$, displaying behaviour largely differing from conventional prestressing materials. When the material was subjected to cyclic loads, a loss of pre-stress occurred after the first load cycle. In subsequent strain-controlled load-cycles, the pre-stress did not further decrease. The stress-strain curve showed a highly non-linear slope for the first cycle. However, when subsequent cycles were examined, higher stiffness and an almost linear slope were observed. Since the different production process of ribbed bars results in deviations in thermo-mechanical properties [3], the behaviour under the same conditions was of great interest.

Innovative strengthening techniques with Fe-SMA bars are under development at Empa. Analytical models for the stress-strain behaviour of the FeSMA bars are therefore needed for numerical and analytical investigations, which have not been developed so far due to the novelty of Fe-SMA ribbed bars.

In this study, the thermo-mechanical behaviours of ribbed bars with nominal diameter $\mathrm{d}_{\text {nom }} 16 \mathrm{~mm}$ and $12 \mathrm{~mm}$ are examined experimentally. The focus of this study lies on investigating the quasistatic, as well as cyclic behaviours after activation. In addition, analytical models for the stress-strain behaviour are developed that serve as base for analytical and numerical investigations in further studies.

\section{Experiments}

\subsection{Material}

The material was received from the provider in the form of coil-shaped bars with nominal diameter of $16 \mathrm{~mm}$, as well as $12 \mathrm{~mm}$. The rib geometry is in accordance to BS 6447 [11]. The bars were cut to a length of $2.4 \mathrm{~m}$ and afterwards straightened mechanically. Figure 1 illustrates ribbed Fe-SMA bars with diameter $16 \mathrm{~mm}$.

For the functioning of the shape-memory effect, the material was pre-strained in a servo-hydraulic testing machine. The extent of pre-strain was limited by the stroke of the available machine as well as the initial eccentricity of the bars.

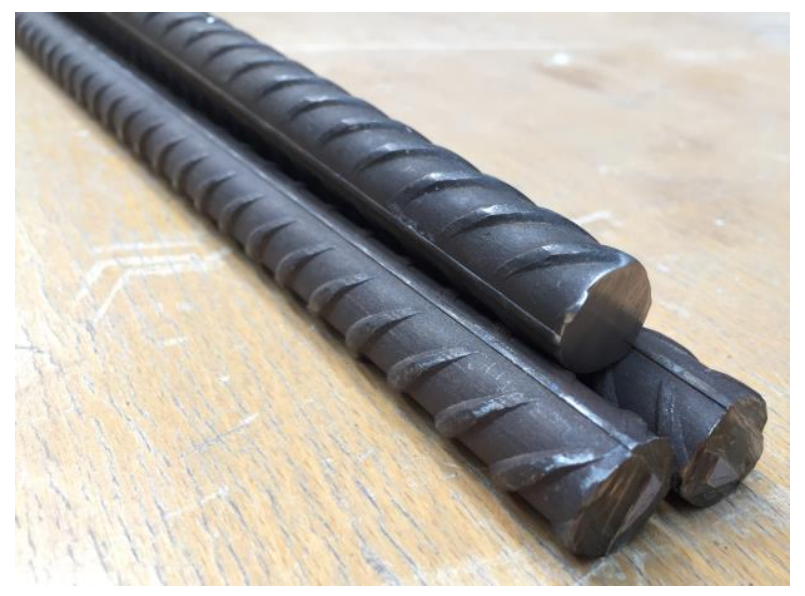

Figure 1. Fe-SMA bars, diameter $16 \mathrm{~mm}$

Specimen and pre-strain levels are listed in Table 1. The process of pre-straining is explained in detail in [9]. The un-loading results in the decrease in strain consisting of an elastic and a pseudoelastic fraction [3]. The sum of these strains was measured at the current specimen between $1.2 \%$ to $1.3 \%$, which is in accordance to [3].

\subsection{Test setup}

Figure 2 shows the test setup prepared for activation. To obtain the stress-strain behaviour in the pre-stressed state, the bars were firstly activated by means of electric current. After cooling, loading was performed by means of a hydraulic jack. Similar setups were previously used in $[3,6]$. The reaction structure consisted of two HEB 360 steel columns and additional stiffening elements rigidly fixed to the strong floor of the laboratory. On the right hand side, a load cell with a load capacity of $400 \mathrm{kN}$ was used to record forces. The left hand side was equipped with a manually actuated hydraulic load jack, which offers a maximum load of $450 \mathrm{kN}$ and maximum stroke of $150 \mathrm{~mm}$. 


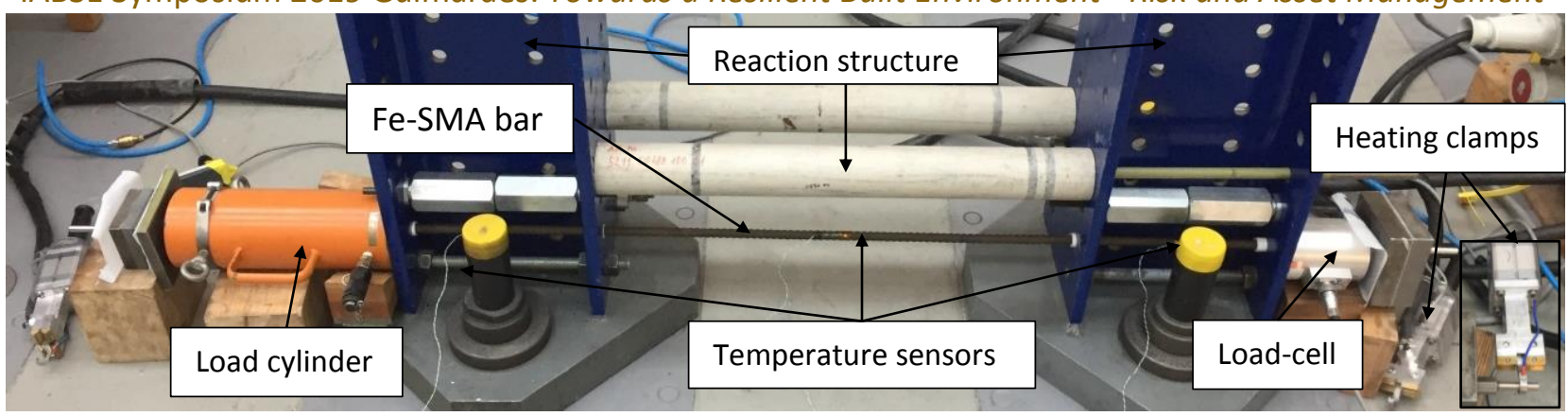

Figure 2. Test setup for activation and tensile testing of Fe-SMA bars

\subsection{Activation}

After pre-straining, threads with the geometry of M15x1.5 were cut onto the diameter $16 \mathrm{~mm}$ ends to enable rigid fixation. The diameter $12 \mathrm{~mm}$ bars were fixed with draw-in wedges. The Fe-SMA bars were activated by resistive heating, meaning increase in temperature by application of an electric current. A heating device was made available by the provider where electricity was applied by means of two clamps at the bar-ends, illustrated in Figure 2. To electrically isolate the sample against all metallic parts, Teflon elements were placed between the Fe-SMA bar and adjacent parts.

Before activation, a small pre-load was applied mechanically by tightening the threads in order to maintain rigid fit during the thermal expansion phase of the alloy. Previous investigations [6] have shown, that a mechanical pre-load to the current extent does not have a significant impact on the final recovery stress. The heating parameters were designed with a maximum power of $3000 \mathrm{kWs}$ and a current of $400 \mathrm{~A}$. The bars were actively heated for approximately 40 to 60 seconds until the target temperature of $190{ }^{\circ} \mathrm{C}$ was reached. The temperature of the Fe-SMA bars was monitored by three thermocouples of Type $K$ at different locations on the bar. Forces were recorded by a load cell. Due to the delay caused by circuit design of the heating device, accurate targeting of a maximum temperature implies some difficulties, but was improved throughout testing. Therefore, the samples show slightly differing activation temperatures, which led to a variation in recovery stresses.

After the sample was cooled down to room temperature, the relaxation behaviour was monitored during subsequent hours.

\subsubsection{Re-activation}

After the first loading of the activated Fe-SMA bars had been performed without failure, the bars were again rigidly fixed and then activated for a second time. The phenomenon of re-activation can be explained by the fact that during loading, the material effectively undergoes a second prestraining process, which results in a generation of Martensite in the alloy.

\subsection{Tensile tests}

\subsubsection{Quasi static loading after activation}

The tensile tests were performed while the FeSMA bar was under pre-stress due to activation. On the center part of the bar, a LVDT was attached to measure the elongation. The maximum load level of the diameter $16 \mathrm{~mm}$ specimen was limited by the reduced crosssection in the threaded fixation.

\subsubsection{Cyclic loading after activation}

To test the material under realistic conditions, a stress-controlled procedure was applied, simulating cyclic loads such as heavy traffic loads on bridge structures. In order to simplify manual control of the process due to absence of electronic control, two groups with each 10 load cycles were applied. In the first group, a stress increase of $+80 \mathrm{MPa}$, and in the second group an increase of $+100 \mathrm{MPa}$ from recovery stress level was targeted. As a result, the differential load between minimum and maximum was slightly increased in every load cycle and considered in the analysis. 


\section{Analytical modelling}

\subsection{Overview}

Aim of the analytical modelling was to find a representative model for the stress-strain behaviour of the Fe-SMA material. Material tests with diameter $16 \mathrm{~mm}$ bars were used for validation. Due to the high non-linearity and different boundary conditions, the model was separated in three fundamental situations:

1. Quasi-static loading in initial state, before activation

2. Quasi-static loading after activation

3. Cyclic loading after activation (subject of current research)

\subsection{Approach}

The Ramberg-Osgood non-linear material model served as base for the analytical description. The model was developed at NASA in 1943 [12] and modified by Rasmussen in 2003 [13]. Due to the pronounced change in slope in the graph of stressstrain behaviour, each model was separated in two parts. Besides the input parameters, which can easily be obtained from tensile test data, exponents $n, m$ and $j$ have to be fitted to the current material. For model 1 , the equation given in [13] was directly applied and delivered accurate representation. Model 2 was modified according to the situation.

\subsection{Model for quasi-static loading in initial state, before activation}

All physical input parameters for the model can be obtained by a standard tensile test. The parameters are illustrated in Figure 3 and consist of the following:

1. Engineering stress as independent variable $\sigma$

2. $0.2 \%$ proof-stress $\sigma_{0.2}$

3. Initial secant modulus for linear portion of the stress-strain curve $E_{0}$

4. Strain at $0.2 \%$ proof-stress $\varepsilon_{0.2}$

5. Tangent modulus at $0.2 \%$ proof-stress $\mathrm{E}_{\mathrm{t} 0.2}$

6. Ultimate stress $\sigma_{\mathrm{u}}$

7. Ultimate strain $\varepsilon_{\mathrm{u}}$

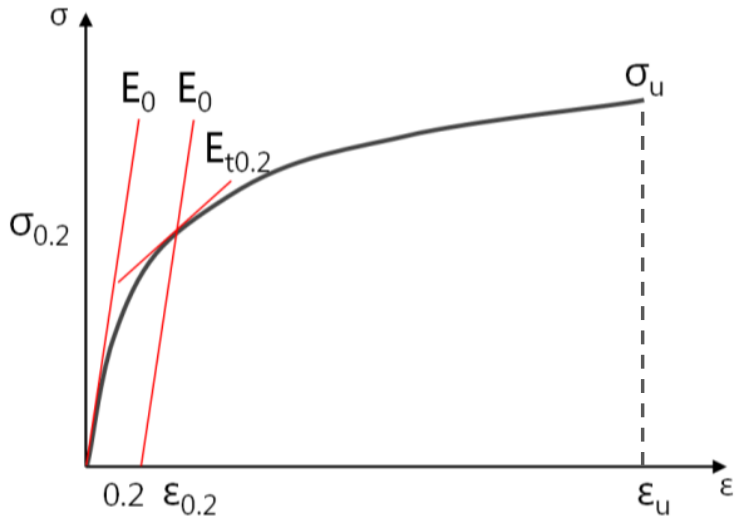

Figure 3. Illustration of input parameters, model quasi-static loading in initial state

The model was formulated as:

- For $\sigma \leq \sigma_{0.2}$ :

$$
\varepsilon(\sigma)=\frac{\sigma}{E_{0}}+0.002 \cdot\left(\frac{\sigma}{\sigma_{0.2}}\right)^{n}
$$

- $F o r \sigma>\sigma_{0.2}$ :

$$
\varepsilon(\sigma)=\frac{\sigma-\sigma_{0.2}}{E_{t 0.2}}+\varepsilon_{u} \cdot\left(\frac{\sigma-\sigma_{0.2}}{\sigma_{u}-\sigma_{0.2}}\right)^{m}+\varepsilon_{0.2}
$$

\subsection{Model for quasi-static loading after activation}

From previous investigations $[6,7]$ with $\mathrm{Fe}$ SMA strips it is known, that the stress-strain curve of activated Fe-SMA bars will approach and then follow the non-activated stressstrain curve. Data shows, that the point of approach is situated at a slightly increased strain from maximum pre-strain. The value of recovery stress acts as an input variable and starting point for the first part of the model. The second part spans between the connection point and the point of failure. The input parameters are depicted in Figure 4.

- For $\sigma \leq \sigma_{j o i n}$ :

$$
\begin{gathered}
\varepsilon(\sigma)=\frac{\sigma-\sigma_{r e c}}{E_{p 0}}+\varepsilon_{\text {join }} \cdot\left(\frac{\sigma-\sigma_{\text {rec }}}{\sigma_{\text {join }}-\sigma_{\text {rec }}}\right)^{j}+(3) \\
\varepsilon_{\text {pre,res }}
\end{gathered}
$$

- For $\sigma>\sigma_{\text {join }}$ :

$$
\begin{aligned}
\varepsilon(\sigma)= & 4.803 \cdot 10^{-6} \cdot \sigma^{2}- \\
& 6.02151 \cdot 10^{-3} \cdot \sigma+ \\
& 1.88108+\varepsilon\left(\sigma_{\text {join }}\right)
\end{aligned}
$$




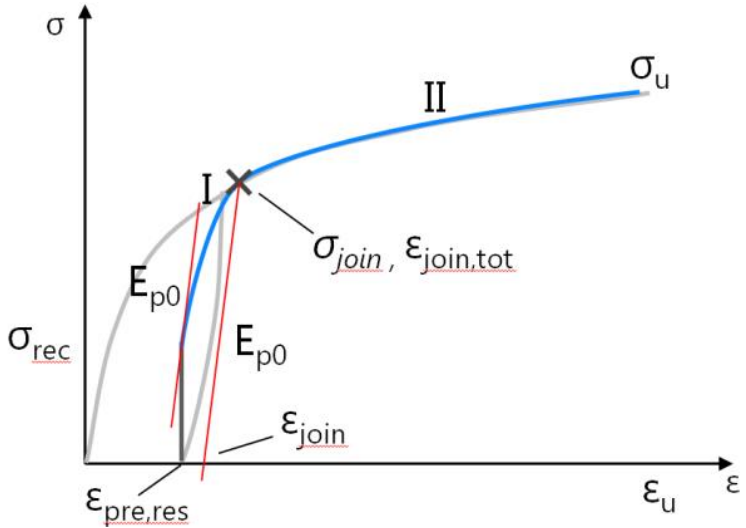

Figure 4. Illustration of input parameters, model quasi-static loading after activation

\section{Results and discussion}

\subsection{Activation and recovery stress}

Figure 5 depicts the first activation process. The Fe-SMA with diameter $12 \mathrm{~mm}$ (d12-1) exhibited excellent recovery stress behaviour $\left(\sigma_{\text {rec }}\right.$ in Table 1 ), equal to the diameter $16 \mathrm{~mm}$ (d16-1-3) bars.

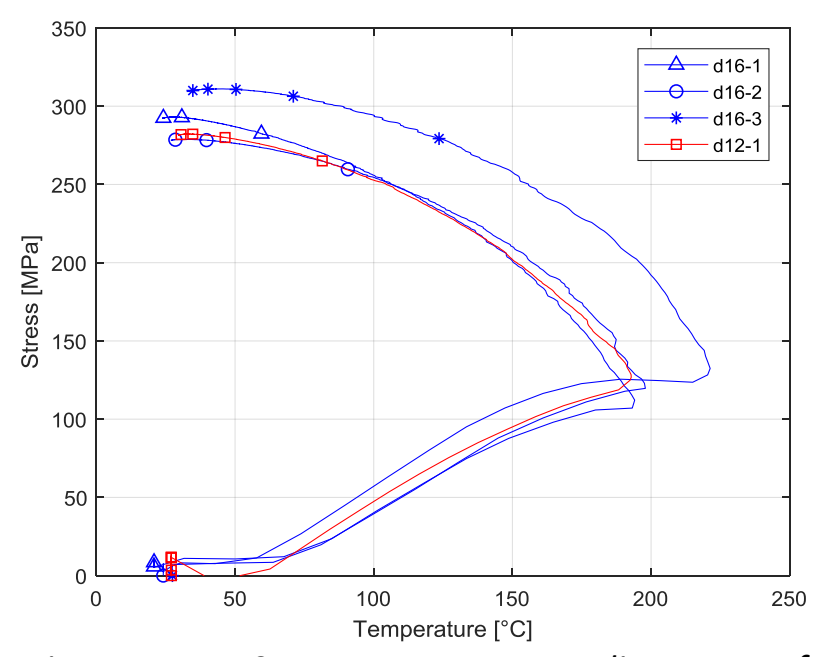

Figure 5. Stress-temperature diagram of activation process

\subsection{Stress relaxation}

Figure 6 depicts the relative decrease in recovery stress $\Delta \sigma$ over time, which is referred to as stressrelaxation. The measurements show a time dependent behaviour, where the highest rate of decrease occurs in the first 6 hours after peak stress, which was also observed in [3]. The obvious inconsistencies in the trends can be explained by ambient temperature variations and have been considered in the evaluation.

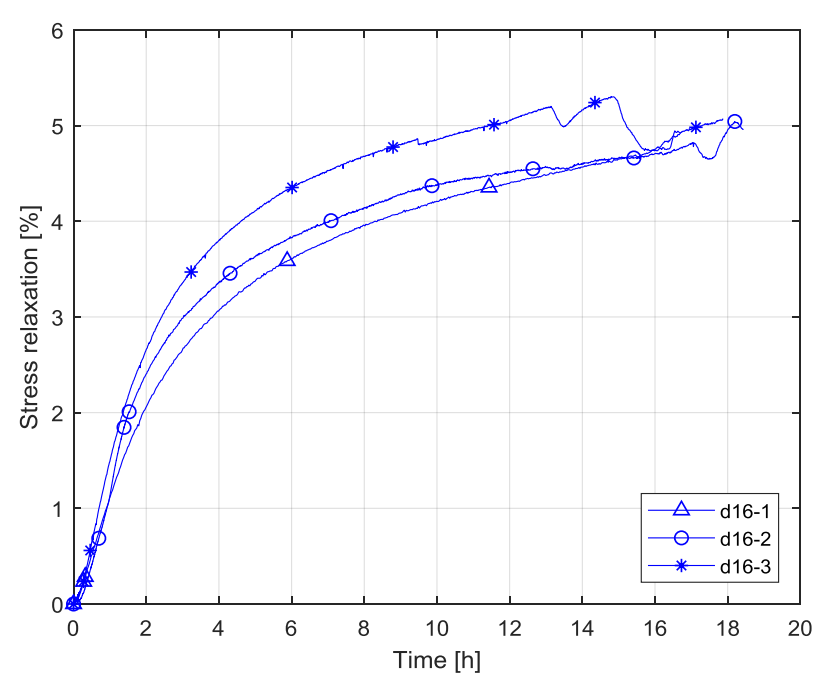

Figure 6. Stress relaxation from peak recoverystress after activation, diameter $16 \mathrm{~mm}$ bars

In [3], the relaxation behaviour of diameter $16 \mathrm{~mm}$ Fe-SMA bars from the same material batch was investigated. Samples were pre-strained to $6.5 \%$ and heated to $200{ }^{\circ} \mathrm{C}$, resulting in a recovery stress of $316 \mathrm{MPa}$. A stress decrease of $19 \mathrm{MPa}$ or $6 \%$ was observed after 10 hours from reaching peak stress. In the current investigations, relaxations between $4 \%$ and $5 \%$ were measured after 10 hours. The data indicates a slight dependency of stress relaxation on recovery stress, however this hypothesis cannot be confirmed at this point and will be subject of further studies.

\subsection{Quasi-static behaviour after activation}

In Figure 7, results of tensile tests after activation (dxx-1-3) and before activation ( $d x x$ ini.) are compared. The offset in data from tensile tests after activation originates from the residual strain $\varepsilon_{\mathrm{p}, \text { res }}$ in the pre-straining process. For samples 1-3 (diameter $16 \mathrm{~mm}$ ), the loading test was stopped at $\sigma_{\max }$ before failure, so that the sample could be reactivated. Therefore, only an estimation for the point of approach to the initial tensile test curve can be provided. The load level for the $12 \mathrm{~mm}$ sample was further increased, so the approaching point could be observed. The test results are listed in Table 1. The material shows similar behaviour to Fe-SMA strips in terms of stiffness and point of approach. Based on data from the authors in [6], the loading curve approaches the initial curve at a relative strain increase of approximately $6.5 \%$ 
from the pre-straining point. The $12 \mathrm{~mm}$ sample of the current study shows a point of approach at relative strain increase of $6 \%$ from pre-straining, which illustrates good accordance. The projected point of approach of diameter $16 \mathrm{~mm}$ samples depicts the same result.

As a representative parameter for stiffness, the secant modulus $E_{s}$ was calculated between $20 \mathrm{MPa}$ and $100 \mathrm{MPa}$ relative to recovery stress level. Values between 62 and $92 \mathrm{GPa}$ were obtained. The same procedure delivers a value of $E_{s}=166 \mathrm{GPa}$ at initial-state tensile tests. Therefore, stiffness decreases significantly when loaded after restrained activation compared to initial loading from zero stress. Influencing parameters are identified as variability in the production process as well as different pre-straining and heating conditions and will be addressed in further studies. In $[5,10]$, a dependency of $E_{s}$ on activation temperature and recovery stress was found, however, no definitive statement can be drawn from data of the current work at this point.

Table 1. Test results

\begin{tabular}{|c|c|c|c|c|}
\hline Sample & 1 & 2 & 3 & 4 \\
\hline $\begin{array}{c}d_{\text {nom }} \\
{[\mathrm{mm}]}\end{array}$ & 16 & 16 & 16 & 12 \\
\hline $\begin{array}{c}\varepsilon_{\mathrm{p}} \\
{[\%]}\end{array}$ & 5.9 & 5.4 & 5.3 & 5.5 \\
\hline $\begin{array}{l}\varepsilon_{\mathrm{p}, \text { res }} \\
{[\%]}\end{array}$ & 4.6 & 4.1 & 4.0 & 4.2 \\
\hline $\begin{array}{l}\mathrm{T}_{\max } \\
{\left[{ }^{\circ} \mathrm{C}\right]}\end{array}$ & 195 & 198 & 221 & 193 \\
\hline $\begin{array}{c}\sigma_{\text {rec }} \\
{[\mathrm{MPa}]}\end{array}$ & 293 & 279 & 311 & 282 \\
\hline $\begin{array}{c}\Delta \sigma(t=10 h) \\
{[\mathrm{MPa}]}\end{array}$ & 12 & 12 & 15 & 11 \\
\hline $\begin{array}{c}E_{s} \\
{[\mathrm{GPa}]}\end{array}$ & 92 & 75 & 62 & 86 \\
\hline $\begin{array}{c}\sigma_{\max } \\
{[\mathrm{MPa}]}\end{array}$ & 610 & 583 & cycl. & 691 \\
\hline
\end{tabular}

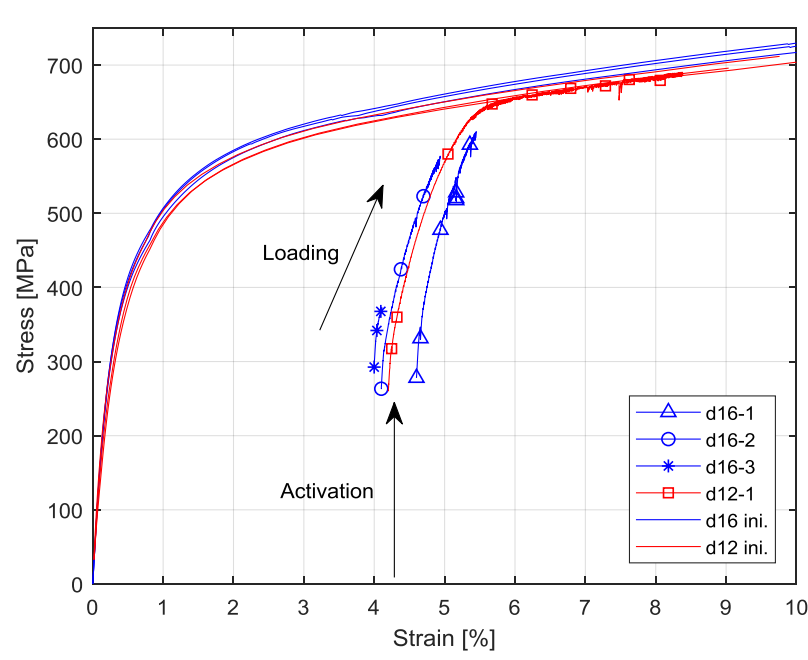

Figure 7. Stress-strain diagram, comparison of tensile test data before and after activation

\subsection{Cyclic behaviour after activation}

Figure 8 shows the stress-strain diagram of cyclic loading of diameter $16 \mathrm{~mm}$ sample number 3 , as well as the positioning of the test relative to the pre straining curve.

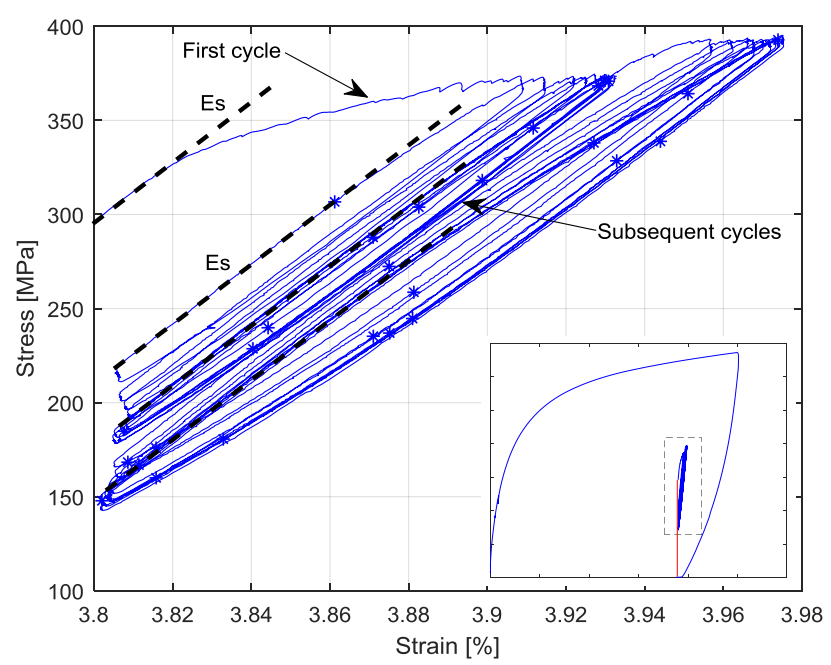

Figure 8. Stress-strain diagram of cyclic loading and global positioning of loading-, relative to prestraining curve

Two groups of load cycles were applied in sequence, firstly increasing the load to an absolute stress of $372 \mathrm{MPa}(+80 \mathrm{MPa})$ in the first group, followed by a level of $392 \mathrm{MPa}(+100 \mathrm{MPa})$. The measurements show that the shape of the first loading curve differs from subsequent cycles. In the first cycle, the material displayed linear behaviour up to a $\Delta \sigma$ of $20 \mathrm{MPa}$, followed by highly non-linear behaviour. In subsequent cycles 
however, a nearly linear ascending branch was apparent between the start of the load cycle and a $\Delta \sigma$ of $100 \mathrm{MPa}$. When secant moduli were compared for the linear sections of the curves, moduli of approximately $144 \mathrm{GPa}$ and $158 \mathrm{GPa}$ were evident for first, and subsequent load-cycles respectively. When the load was increased to $100 \mathrm{MPa}$, the sample exhibited the same behaviour.

It is clear that the differential load increase between each load-cycle due to the testprocedure resulted in an equally decreasing prestress of about $36 \mathrm{MPa}$ in the first group of cycles and $18 \mathrm{MPa}$ in the second one. This circumstance is considered in further current research. Parametric studies will be performed to study the loss of pre-stress due to additional loading with fixed stress boundaries.

\subsection{Modelling}

In Figure 9, generated and experimental data of diameter $16 \mathrm{~mm}$ bars is depicted within the total strain range and until $5 \%$ strain. The following values were used in Equations 1 and 2: $\mathrm{E}_{0}=163300 \mathrm{MPa}, \sigma_{0.2}=396 \mathrm{MPa}, \mathrm{n}=4, \mathrm{E}_{\mathrm{t} 0.2}=35000$ $\mathrm{MPa}, \quad \varepsilon_{\mathrm{u}}=0.3113, \quad \sigma_{\mathrm{u}}=868 \mathrm{MPa}, \quad \varepsilon_{0.2}=0.00443$, $\mathrm{m}=3.6$. Root-mean-square error (RMSE) was calculated equaling $7.04 \mathrm{MPa}$, which displays good alignment.

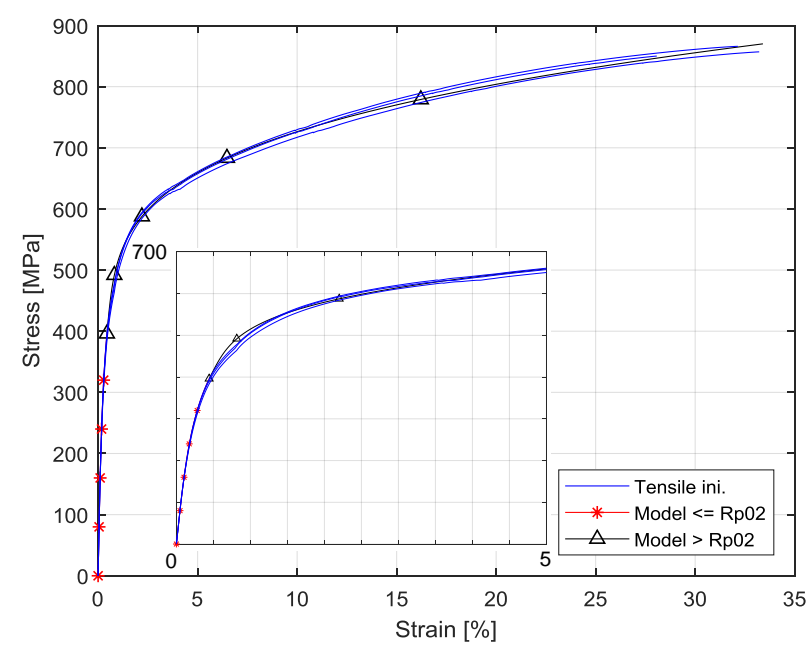

Figure 9. Comparison data and model, quasi-static loading before activation, total strain range and up to $5 \%$

Figure 10 depicts the comparison of material model vs. experimental data of tensile tests after restrained activation. The following values were used in Equations 3 and 4: $\sigma_{\text {rec }}=277.9 \mathrm{MPa}$, $E_{p 0}=150000 \mathrm{MPa}, \varepsilon_{\text {join }}=0.01098, j=2.5$, $\varepsilon\left(\sigma_{\text {join }}\right)=0.03892$. The model starting point adjusts to different recovery stresses. Due to lack of sufficient verification data, a quality of fit parameter will be obtained from further studies.

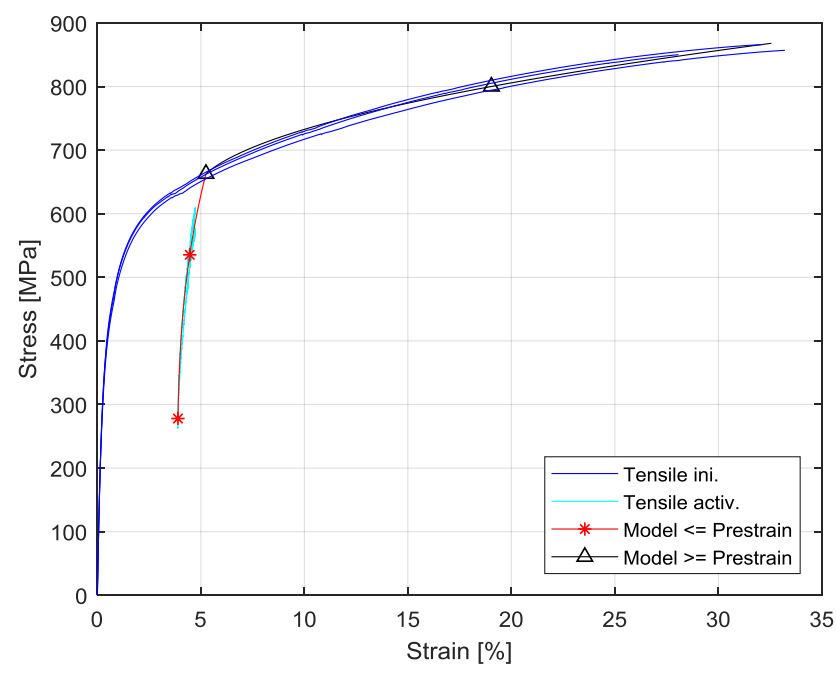

Figure 10. Comparison data and model, quasistatic loading after activation

\section{Conclusions and Outlook}

1. Activation and re-activation of diameter $16 \mathrm{~mm}$ and $12 \mathrm{~mm}$ Fe-SMA bars were successfully performed. The diameter $16 \mathrm{~mm}$ and $12 \mathrm{~mm}$ samples show similar recovery stress in the range of $282 \mathrm{MPa}$.

2. The stress-relaxation behaviour shows a time-dependent rate of stress-reduction. After 10 hours, a stress relaxation between $4 \%$ and $5 \%$ was measured. Based on comparison with data from [3], a dependency of stress relaxation on initial recovery stress is expected and is subject of further research.

3. Quasi-static tensile tests after activation show a lower secant modulus between 62 and $92 \mathrm{GPa}$ than before activation. The dependency of stiffness on recovery stress will be addressed in future studies.

4. Cyclic loading after activation showed different behaviour of the first and subsequent load cycles. In the first cycle, a loss of pre-stress was observed. The first load-cycle shows highly non-linear 
properties, subsequent cycles display large linear sections.

5. Analytical models for the stress-strain behaviour before and after activation show very good alignment with experimental data of diameter $16 \mathrm{~mm}$ bars. Future studies will examine the validity for other Fe-SMA components.

\section{Acknowledgements}

This work has been supported by the Swiss National Science Foundation, Grant No. 200021_175998. The continuous support and supply of materials by re-fer AG is greatly appreciated.

\section{References}

[1] Z. Dong, U. E. Klotz, C. Leinenbach, A. Bergamini, C. Czaderski, and M. Motavalli, "A Novel Fe-Mn-Si Shape Memory Alloy With Improved Shape Recovery Properties by VC Precipitation," Advanced Engineering Materials, vol. 11, no. 1-2, pp. 40-44, 2009.

[2] C. Czaderski, M. Shahverdi, R. Brönnimann, C. Leinenbach, and $M$. Motavalli, "Feasibility of iron-based shape memory alloy strips for prestressed strengthening of concrete structures," Construction and Building Materials, vol. 56, pp. 94-105, 2014.

[3] J. Michels, M. Shahverdi, C. Czaderski, and R. El-Hacha, "Mechanical performance of Fe-SMA ribbed bars for concrete prestressing," accepted for publication in ACI Materials Journal.

[4] M. Shahverdi, C. Czaderski, P. Annen, and M. Motavalli, "Strengthening of RC beams by iron-based shape memory alloy bars embedded in a shotcrete layer," Engineering Structures, vol. 117, pp. 263273, 2016.

[5] M. Shahverdi, C. Czaderski, and $M$. Motavalli, "Iron-based shape memory alloys for prestressed near-surface mounted strengthening of reinforced concrete beams," Construction and
Building Materials, vol. 112, pp. 28-38, 2016.

[6] M. Shahverdi, J. Michels, C. Czaderski, and M. Motavalli, "Iron-based shape memory alloy strips for strengthening RC members: Material behavior and characterization," Construction and Building Materials, vol. 173, pp. 586-599, 2018/06/10/ 2018.

[7] E. Ghafoori, E. Hosseini, C. Leinenbach, J. Michels, and M. Motavalli, "Fatigue behavior of a Fe-Mn-Si shape memory alloy used for prestressed strengthening," Materials \& Design, vol. 133, pp. 349-362, 2017.

[8] W. J. Lee, B. Weber, and C. Leinenbach, "Recovery stress formation in a restrained $\mathrm{Fe}-\mathrm{Mn}$-Si-based shape memory alloy used for prestressing or mechanical joining," Construction and Building Materials, vol. 95, pp. 600-610, 2015.

[9] W. J. Lee, B. Weber, G. Feltrin, C. Czaderski, M. Motavalli, and C. Leinenbach, "Phase transformation behavior under uniaxial deformation of an $\mathrm{Fe}-\mathrm{Mn}-\mathrm{Si}-\mathrm{Cr}-\mathrm{Ni}-\mathrm{VC}$ shape memory alloy," Materials Science and Engineering: A, vol. 581, pp. 1-7, 2013.

[10] W. J. Lee, B. Weber, G. Feltrin, C. Czaderski, M. Motavalli, and C. Leinenbach, "Stress recovery behaviour of an $\mathrm{Fe}-\mathrm{Mn}-\mathrm{Si}-\mathrm{Cr}-\mathrm{Ni}-\mathrm{VC}$ shape memory alloy used for prestressing," Smart Materials and Structures, vol. 22, no. 12, p. 125037, 2013.

[11] BSI, "Stainless steel bars for the reinforcement of and use in concrete Requirements and test methods," British Standards, vol. BS 6744:2001 + A2:2009, p. 28, 2009.

[12] W. Ramberg and W. R. Osgood, "Description of stress-strain curves by three parameters," 1943.

[13] K. J. Rasmussen, "Full-range stress-strain curves for stainless steel alloys," Journal of constructional steel research, vol. 59, no. 1, pp. 47-61, 2003. 